\title{
Uniaxial compressive failure of brittle materials as instability of damaging microcracked solids
}

\author{
Luigi Gambarotta*, Ilaria Monetto \\ Department of Structural and Geotechnical Engineering, University of Genova, Via Montallegro 1, 16145 Genova, Italy
}

Received 16 August 2000; revised and accepted 2 August 2001

\begin{abstract}
Uniaxial compressive failure of brittle materials is modelled as buckling of microcracked solids undergoing damage. The brittle material is modelled as an isotropic linear elastic matrix containing a random distribution of non-interacting microcracks. Increasing the stress level, the material response evolves from isotropy at the natural state to orthotropy in damaged states. Furthermore, the overall stiffness decreases until a critical condition of equilibrium bifurcation may be reached. The related critical value of the compressive stress, provided by the buckling analysis of orthotropic elastic sheets, is then assumed as compressive strength of the brittle material. In order to predict damage evolution and model the incremental response, simplified crack configurations are considered. The obtained theoretical results show the dependence of the compressive strength on the matrix toughness and microcrack parameters, and are in good correlation with the experimental results obtained on compressed specimens constrained between frictionless devices. @ 2002 Éditions scientifiques et médicales Elsevier SAS. All rights reserved.
\end{abstract}

Keywords: Microcracked solids; Damage; Compressive failure; Instability

\section{Introduction}

In the framework of the micromechanical approach to modelling brittle materials subjected to compression, damage is commonly postulated to emanate from inherent microcracks uniformly distributed in an elastic matrix. Several models have been developed to describe crack growth based on the solution for a single sliding crack in an infinite sheet. When uniaxial compression is considered, such models can represent nonlinear inelastic strain inducing dilatancy and cyclic dissipation while a stable microcrack growth is determined (Horii and Nemat-Nasser, 1986). It follows that a limit value of compression to be assumed as compressive strength of the material cannot be obtained. To circumvent this drawback, Li and Lajtai (1998) proposed a non-local limit strength criterion referring to the average stress state at the neighbourhood of the crack tip. On the other hand, several models explicitly based on linear elastic fracture mechanics criteria and taking into account the elastic interaction among microcracks have been developed. To this end, the evolution of proper crack distributions leading to a limit compressive stress and the related splitting failure mode have been considered (Ashby and Hallam, 1986; Myer et al., 1992; Gambarotta, 1993). Nevertheless, a strong sensitivity of the model response on the assumed crack pattern due to possible shielding effects has been observed, so that in some cases a stable crack growth has been detected (Kemeny and Cook, 1987; Kachanov, 1992).

Moreover, the presence of dominant compressive stress states suggests the possibility of failure modes corresponding to local and global instabilities induced by the decreasing of stiffness due to the damage process. This is the point of some contributions found in literature starting from the pioneering paper by Biot (1965), who first proposed that compression failure involves internal instabilities of a solid and sought for elastic solutions with reference to undamaged materials. Bazant (1967) analysed

\footnotetext{
* Correspondence and reprints.

E-mail address: gambarotta@ diseg.unige.it (L. Gambarotta).
} 
the stability of compressed solids made orthotropic by damage evolution. Yet, the notion that instability affects fracture of a compressed specimen weakened by axial cracks was clearly established by Kendall (1978), who studied the splitting failure mode on the basis of simplified structural schemes. Buckling is also one of the principal aspects of the crack band model by Bazant and Xiang (1997), focusing on the size effects in compression fracture.

In this frame, an equilibrium bifurcation approach to the compressive failure of microcracked solids is here considered. Damage evolution of uniaxially compressed elastic sheets is expected to decrease the elastic solid stiffness until a critical condition of equilibrium bifurcation is reached. The critical stress so provided is assumed as compressive strength of the brittle material modelled as an isotropic linear elastic matrix containing a random distribution of non-interacting microcracks. The formulation consists of three phases. First, damage evolution of the microcracked elastic solid is predicted. As an example, in Section 2 the equivalent crack model developed by Gambarotta (1993) is used to predict crack growth in the brittle material under uniaxial compression. Secondly, approximations for the incremental elastic moduli of the orthotropic damaged solid are derived. Section 3 lays out such a derivation here based on a simplified constitutive model for the incremental response. Lastly, the stability analysis of the uniformly compressed sheet undergoing damage is carried out. As discussed in Section 4 , local bifurcations are derived by the loss of ellipticity of the field equations, while the overall bifurcation conditions are obtained by the existence of adjacent configurations in equilibrium (Kerr and Tang, 1967). On this basis, the theoretical strength is regarded as the compressive stress at which a critical condition of bifurcation is attained for the related damage level. The approach is described in Section 5 where the sensitivity of the theoretical compressive strength to model parameters is also analysed. The paper is addressed to get a more comprehensive interpretation of the phenomenon under consideration rather than on providing quantitative estimates of practical use for the compressive strength. Particular attention is focused on possible effects of the specimen geometry as well as the onset of localized deformations, which the stability analysis permits us to take into account. Nevertheless, the results show surprisingly that shape independent theoretical compressive strengths can be obtained for a wide range of model parameters.

As discussed in Section 3, the complexity of the rigorous approach for the derivation of incremental moduli required by the stability analysis justifies some restrictive simplifications introduced to make the analysis be tractable. The most substantial one may be to neglect interaction between microcracks (Myer et al., 1992). On the other hand, it is also known that this hypothesis can provide accurate results for randomly located and oriented crack arrays, as shown by numerical experiments carried out by Kachanov (1992) and Zhan et al. (1999) and discussed theoretically by Alpa and Gambarotta (1993) as well. Furthermore, according to Kachanov's (1992) and Zhan et al.'s (1999) analyses, the approximation of non-interacting cracks still provides slightly different but qualitatively good results for two dimensional arrays of randomly located parallel cracks. Note that the last ones correspond to limit crack configurations which are representative of the material in orthotropically damaged states, as assumed in the incremental analysis.

\section{Damage evolution in uniaxially compressed brittle materials}

The two dimensional modelling of the brittle material considers an isotropic linear elastic matrix containing a random distribution of non-interacting straight microcracks having equal length $2 c$ and random orientation $\vartheta_{n}\left(\left|\vartheta_{n}\right| \leqslant \pi / 2\right)$ with respect to the $x_{1}$ axis (Fig. 1a). Due to an increasing uniaxial compression $\sigma_{22}$ applied in the $x_{2}$ direction, progressive sliding and non-planar extension of the pre-existing flaws occur causing damage.

In the approximation of non-interacting microcracks, damage evolution can be predicted regarding each crack as an isolated one contained in an infinite linear elastic, isotropic and homogeneous sheet. Let $\sigma_{n}$ and $\tau_{n}$ be the resolved stresses induced by far-field stresses on the crack plane of unit normal $\boldsymbol{n}$. Being as $\sigma_{n}$ is always negative, the flaw is closed and the shear stress $\tau_{n}$ tends to make the opposite faces in contact slide depending on the active frictional resistance. Regarding frictional stresses as uniformly distributed across the opposite faces, only the pre-existing flaws with initial angle $\left|\vartheta_{n}\right|>\arctan \mu$ ( $\mu$ is the friction coefficient) experience sliding, the other cracks being locked by friction. Only a non-zero mode II Stress Intensity Factor (SIF) $K_{\mathrm{II}}$ is induced at both tips of each sliding crack by the effective shear stress $\tau_{n \mathrm{e}}=\tau_{n}+\mu \sigma_{n}$. Under increasing compression two wing cracks symmetrically develop from the tips of the active initial flaws and while propagating align themselves with the direction of the dominant principal compression (Brace and Bombolakis, 1963). The symmetry of the overall response, changing from isotropic for the material in the natural state to orthotropic in damaged states, results from assuming a symmetric crack distribution with respect to the axis $x_{2}$ (for any $\vartheta_{n}$-oriented crack a symmetric one is considered).

With reference to Fig. 1a, let $l_{n}$ be the actual extension of wing branches for $\vartheta_{n}$-oriented pre-existing flaws that are obtained at any step of the compressive loading process. Then, the mean total extension length normalised with respect to the initial flaw length $2 c$ is here assumed as a measure of the damage:

$$
\zeta=\frac{1}{\pi} \int_{\Omega} \frac{l_{n}}{c} \mathrm{~d} \vartheta,
$$




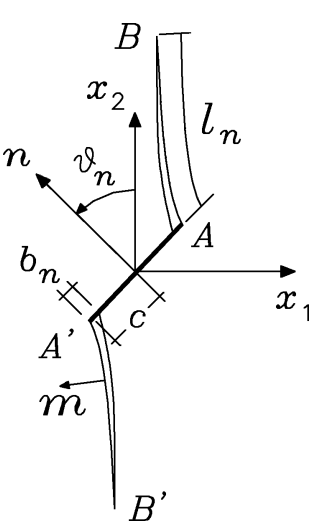

(a)

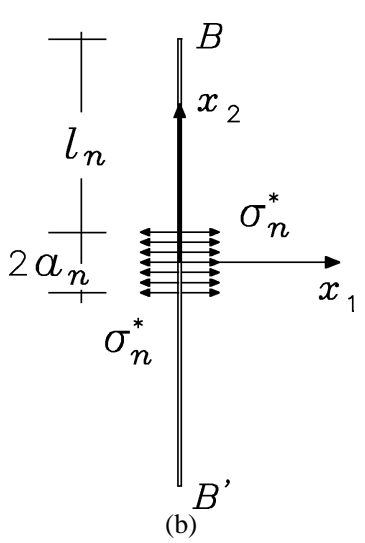

(b)

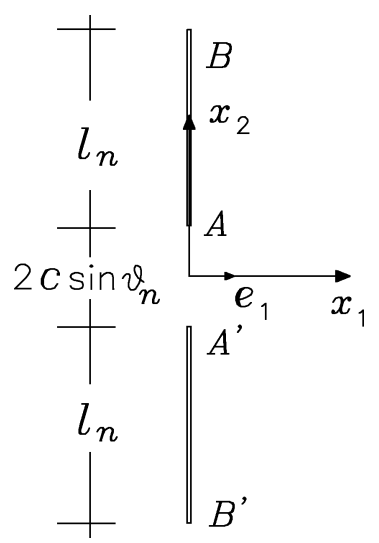

(c)

Fig. 1. (a) $\boldsymbol{n}$-oriented actual crack; (b) equivalent crack for predicting damage evolution; (c) simplified configuration for the incremental analysis.

where the integral is over all orientations in the range $\Omega \equiv[-\pi / 2, \pi / 2]$, but only active cracks give an effective contribution. $\zeta=0$ represents the material in the natural state, whereas a non-zero damage parameter $\zeta>0$ characterizes a damaged configuration. In fact, a one-to-one correspondence of $\zeta$ on the applied stress $\sigma_{22}$ can be proved to exist as a consequence of the same correspondence of the crack extension on the applied uniaxial compression for each orientation.

More explicit results require now to derive $l_{n}$ from the analysis of crack growth in the brittle material under uniaxial compression. Note that the so-called sliding crack problem has already been analysed in detail. The exact formulation of the related two dimensional boundary value problem was first given by Horii and Nemat-Nasser (1985), who developed a numerical solution for curved crack growth. On the other hand, only either regarding the wing cracks as straight lines or introducing the concept of equivalent crack, analytical approximate estimates of the SIF have been obtained (Zaitsev and Wittmann, 1981; Steif, 1984; Ashby and Hallam, 1986; Horii and Nemat-Nasser, 1986; Fanella and Krajcinovic, 1988).

As an example, according to the equivalent crack model proposed by Gambarotta (1993), each set of equi-oriented kinked cracks is replaced by an equivalent one. As shown in Fig. $1 \mathrm{~b}$ for the $\vartheta_{n}$-oriented set, equivalent cracks are straight, aligned with the direction of the compressive stress, and subjected to a distribution of uniform tractions $\sigma_{n}^{*}$ on the initial crack lengths $2 a_{n}$. The parameters $a_{n}$ and $\sigma_{n}^{*}$ are defined on the basis of the equivalence criterion at the onset of fracture $\left(l_{n} \approx 0\right)$ and depend explicitly on the initial flaw length and orientation and also on the stress level through the effective shear stress:

$$
a_{n}=\frac{3}{4} c \cos ^{2} \vartheta_{n}, \quad \sigma_{n}^{*}=\frac{4}{3} \frac{\tau_{n \mathrm{e}}}{\cos \vartheta_{n}} .
$$

Through the solution given by Irwin (1957) (Tada et al., 1973), the SIF $K_{\mathrm{I}}^{\mathrm{e}}$ at the tips of the equivalent cracks yields:

$$
K_{\mathrm{I}}^{\mathrm{e}}=\frac{2}{\sqrt{\pi}} \sqrt{\left(a_{n}+l_{n}\right)} \sigma_{n}^{*} \arcsin \left(\frac{a_{n}}{a_{n}+l_{n}}\right),
$$

which is assumed to be an estimate of the SIF at the tips of the kinked cracks. At any step of the compressive loading process, wing crack lengths $l_{n}$ can then be obtained by equating equation (3) with the fracture toughness $K_{\text {IC }}$ of the brittle matrix. Note that for each set of $\boldsymbol{n}$-oriented pre-existing flaws the response to uniaxial compression is stable $\left(\partial K_{\mathrm{I}}^{\mathrm{e}} / \partial l_{n}<0\right)$ and a one-toone correspondence of the crack extension length $l_{n}$ on the compressive stress level $\sigma_{22}$ exists. The damage evolution is then predicted uniquely by the mentioned model.

\section{Incremental constitutive response of uniaxially compressed brittle materials}

Regarding the damage as a uniform field and referring to a representative element large enough with respect to the embedded cracks, frictional sliding along the pre-existing flaws and sliding - induced opening of the wing branches may be treated as inelastic contributions to the macroscopic deformation. It is worth noting that such a model is not valid during the post-failure phase, in which experimental evidences show that macrocracking or localised cracked bands extensively occur. In the framework 
of the kinematic analysis of heterogeneous materials (Nemat-Nasser and Hori, 1993), the inelastic strain contribution $\boldsymbol{\varepsilon}^{\mathrm{i}}$ may be written in the following form:

$$
\boldsymbol{\varepsilon}^{\mathrm{i}}=\frac{N}{\pi} \int_{\Omega} \int_{-L_{n}}^{L_{n}} \operatorname{sym}([\boldsymbol{u}] \otimes \boldsymbol{m}) \mathrm{d} \xi \mathrm{d} \vartheta
$$

where $N$ is the number of cracks per unit area and $[\boldsymbol{u}]$ is the displacement jump across the opposite faces of the crack path, having total length $2 L_{n}\left(L_{n}=c+l_{n}\right)$ and unit normal vector $\boldsymbol{m}$ referred to equi-oriented flaws (Fig. 1a). The total strain is then given by:

$$
\boldsymbol{\varepsilon}=\boldsymbol{\varepsilon}^{\mathrm{e}}+\boldsymbol{\varepsilon}^{\mathrm{i}}=\mathbf{D} \boldsymbol{\sigma}+\frac{N}{\pi} \int_{\Omega} \int_{-L_{n}}^{L_{n}} \operatorname{sym}([\boldsymbol{u}] \otimes \boldsymbol{m}) \mathrm{d} \xi \mathrm{d} \vartheta,
$$

where $\boldsymbol{\varepsilon}^{\mathrm{e}}$ is the elastic strain and $\mathbf{D}$ is the fourth-order compliance tensor of the elastic matrix.

For each set of parallel microcracks, the displacement jump $[\boldsymbol{u}]$ across crack faces also depends on sliding $b_{n}$ and wing crack length $l_{n}$ (Fig. 1a) which may be assumed as internal variables to which thermodynamic conjugate forces are associated. Since such variables are given by evolution equations, the general approach to the analysis of uniaxially compressed microcracked sheets must be formulated in incremental form. In this frame the incremental inelastic strain $\dot{\boldsymbol{\varepsilon}}^{\mathrm{i}}\left(\vartheta_{n}\right)$ related to the set of $\vartheta_{n}$ oriented flaws may be written as follows:

$$
\dot{\boldsymbol{\varepsilon}}^{\mathrm{i}}\left(\vartheta_{n}\right)=\mathbf{D}^{\mathrm{i}}\left(\vartheta_{n}\right) \dot{\boldsymbol{\sigma}}+\boldsymbol{\varepsilon}_{b}\left(\vartheta_{n}\right) \dot{b}+\boldsymbol{\varepsilon}_{l}\left(\vartheta_{n}\right) \dot{l}=\mathbf{D}_{\mathbf{t}}^{\mathrm{i}}\left(\vartheta_{n}\right) \dot{\boldsymbol{\sigma}}
$$

where $\mathbf{D}^{\mathrm{i}}\left(\vartheta_{n}\right)$ is the symmetric fourth-order compliance tensor providing the incremental inelastic strain due to the stress increment $\dot{\boldsymbol{\sigma}}$, being crack sliding and extension locked, and $\boldsymbol{\varepsilon}_{b}\left(\vartheta_{n}\right), \boldsymbol{\varepsilon}_{l}\left(\vartheta_{n}\right)$ are the incremental inelastic strains due to a unit sliding and to a unit extension, respectively. During the loading process the rates of crack sliding $\dot{b}$ and extension $\dot{l}$ are obtained with reference to two limit states corresponding to sliding and damage activation (Moss and Gupta, 1982; Basista and Gross, 1998), under the constraints provided by the unilateral contact condition between the opposite faces of the wing cracks (that implies $\left.b_{n} \geqslant 0\right)$ and the irreversibility condition of crack evolution $(i \geqslant 0)$. Since crack sliding and growth increments may be expressed in terms of the stress rates $\dot{\sigma}$, the right side of equation (6) states the constitutive equation by means of the incremental fourth-order compliance tensor $\mathbf{D}_{\mathrm{t}}^{\mathrm{i}}\left(\vartheta_{n}\right)$. Complex forms for $\mathbf{D}_{\mathrm{t}}^{\mathrm{i}}\left(\vartheta_{n}\right)$ have been derived at different levels of detail by Moss and Gupta (1982), Nemat-Nasser and Obata (1988) and more recently by Basista and Gross (1998). On the other hand, the mentioned formulations are referred to principal stress states in order to describe the dilatancy of brittle materials induced by crack growth aligned with the dominant compression; yet, general formulations extending the model response to shearing stresses, as required by the stability analysis, do not seem to be available. Thus, mainly due to the prescriptions on the sign of the internal variables, the complexity of the rigorous approach and the lack of a shearing model for the microcracked solid suggest considering a simplified approach to the incremental constitutive response. Based on such considerations, the simplifying assumption of neglecting both crack sliding and extension $(\dot{b}=0, \dot{l}=0)$ is put forward, so that the instantaneous compliance tensor $\mathbf{D}_{\mathrm{t}}^{\mathrm{i}}\left(\vartheta_{n}\right)$ is approximated by the secant one $\mathbf{D}^{\mathrm{i}}\left(\vartheta_{n}\right)$.

In order to derive explicit results, the simplified crack configuration shown in Fig. 1c is used for the computation of the inelastic rate contribution to compliances. Two collinear cracks of equal length are aligned with the axis $x_{2}$; each one of them corresponds to a branch of the kinked crack, whereas the initial flaw, which is assumed to be closed and inactive $(\dot{b}=0)$, is replaced by the ligament $A A^{\prime}$. The second assumption of stationary cracks in an incremental loading step $(i=0)$ leads us to consider the crack length equal to $l_{n}$ predicted in the damage evolution analysis. The inelastic contribution $\dot{\boldsymbol{\varepsilon}}^{\mathrm{i}}$ to the incremental mean strain $\dot{\boldsymbol{\varepsilon}}$ is then given by:

$$
\dot{\boldsymbol{\varepsilon}}^{\mathrm{i}}=\frac{2 N}{\pi} \int_{\Omega} \operatorname{sym}\left(\langle[\dot{\boldsymbol{u}}]\rangle \otimes \boldsymbol{e}_{1}\right) l_{n} \mathrm{~d} \vartheta
$$

where $\langle[\dot{\boldsymbol{u}}]\rangle$ is the mean displacement discontinuity increment across the opposite faces of each crack having unit normal vector $\boldsymbol{e}_{1}$. It is worth noting that only the contributions $\dot{\varepsilon}_{11}^{\mathrm{i}}$ and $\dot{\varepsilon}_{12}^{\mathrm{i}}$ related to the lateral and shear strains, respectively, are not vanishing:

$$
\dot{\varepsilon}_{11}^{\mathrm{i}}=\frac{2 N}{\pi} \int_{\Omega}\left\langle\left[\dot{u}_{1}\right]\right\rangle l_{n} \mathrm{~d} \vartheta, \quad \dot{\varepsilon}_{22}^{\mathrm{i}}=0, \quad \dot{\varepsilon}_{12}^{\mathrm{i}}=\frac{N}{\pi} \int_{\Omega}\left\langle\left[\dot{u}_{2}\right]\right\rangle l_{n} \mathrm{~d} \vartheta .
$$


Here $\left\langle\left[\dot{u}_{1}\right]\right\rangle$ and $\left\langle\left[\dot{u}_{2}\right]\right\rangle$ denote the increments of the mean displacement jumps in the $x_{1}$ and $x_{2}$ directions, which can be written as follows by an application of the virtual work principle:

$$
\left\langle\left[\dot{u}_{1}\right]\right\rangle=\frac{2 \alpha}{E l_{n}} \int_{0}^{l_{n} / 2}\left[\widehat{K}_{\mathrm{I} A}^{2}(\xi)+\widehat{K}_{\mathrm{I} B}^{2}(\xi)\right] \mathrm{d} \xi \dot{\sigma}_{11}, \quad\left\langle\left[\dot{u}_{2}\right]\right\rangle=\frac{2 \alpha}{E l_{n}} \int_{0}^{l_{n} / 2}\left[\widehat{K}_{\mathrm{II} A}^{2}(\xi)+\widehat{K}_{\mathrm{II} B}^{2}(\xi)\right] \mathrm{d} \xi \dot{\sigma}_{12},
$$

where $E$ is the Young modulus and $v$ the Poisson ratio of the matrix ( $\alpha=1-v^{2}$ for plane strain and $\alpha=1$ for plane stress). In equations (9) $\widehat{K}_{Z A}(\xi)$ and $\widehat{K}_{Z B}(\xi)$ denote the Mode Z (Z=I, II) SIFs at tips $A$ and $B$ of two collinear cracks like the ones in Fig. 1c having size $2 \xi$ and distance $\left[l_{n}+2 c \sin \vartheta_{n}\right]$ between their midpoints, and subjected to uniform distributions of unit normal and shear stresses acting on their faces. Substituting in equations (9) the SIFs given by Erdogan (1962) (Tada et al., 1973), the increments of the mean displacement jumps follow:

$$
\left\langle\left[\dot{u}_{i}\right]\right\rangle=\frac{2 \alpha}{E l_{n}} \int_{0}^{l_{n} / 2}\left[g_{A}(\xi)+g_{B}(\xi)\right] \mathrm{d} \xi \dot{\sigma}_{1 i} \quad(i=1,2),
$$

where:

$$
\begin{aligned}
& g_{A}(\xi)=\frac{\pi}{2 \beta k^{2}}\left(l_{n}+2 c \sin \vartheta_{n}+2 \xi\right)\left[\frac{\mathrm{E}(k)}{\mathrm{K}(k)}-\beta^{2}\right]^{2}, \quad g_{B}(\xi)=\frac{\pi}{2 k^{2}}\left(l_{n}+2 c \sin \vartheta_{n}+2 \xi\right)\left[1-\frac{\mathrm{E}(k)}{\mathrm{K}(k)}\right]^{2} \\
& \beta=\frac{l_{n}+2 c \sin \vartheta_{n}-2 \xi}{l_{n}+2 c \sin \vartheta_{n}+2 \xi}, \quad k=\sqrt{1-\beta^{2}}
\end{aligned}
$$

and $\mathrm{K}(k)$ and $\mathrm{E}(k)$ are complete elliptic integrals of the first and second kind, respectively.

When the crack extension $l_{n}$ for each orientation is evaluated as a function of the applied stress, the incremental inelastic strain components may be obtained by using equations (8) and (10)-(12). They can be expressed synthetically in the form:

$$
\dot{\varepsilon}_{11}^{\mathrm{i}}=\mathcal{A} \dot{\sigma}_{11}, \quad \dot{\varepsilon}_{12}^{\mathrm{i}}=\frac{\mathcal{A}}{2} \dot{\sigma}_{12},
$$

being:

$$
\mathcal{A}=\eta \frac{\alpha}{E} \mathcal{F}\left(\mu, \frac{\sigma_{22} \sqrt{\pi c}}{K_{\mathrm{IC}}}\right),
$$

which shows their dependence on the model parameters. It can be proved that $\eta$ in equation (14) is related to the initial microcrack density $N c^{2}$; however, the restrictive simplifications at the basis of the present model suggest not directly correlating macroscopic response with microstructure in the way considered in the analysis, so that $\eta$ may be regarded as a parameter to be calibrated later by the comparison with experimental data.

To conclude, the incremental stress-strain relation yields

$$
\dot{\boldsymbol{\varepsilon}}=\left(\mathbf{D}+\mathbf{D}^{\mathrm{i}}\right) \dot{\boldsymbol{\sigma}}=\mathbf{D}^{*} \dot{\boldsymbol{\sigma}}
$$

where the non-vanishing components of the tensor $\mathbf{D}^{\mathrm{i}}$ are:

$$
D_{1111}^{\mathrm{i}}=\mathcal{A} \quad \text { and } \quad D_{1212}^{\mathrm{i}}=\frac{1}{4} \mathcal{A},
$$

and depend on the elastic moduli $E$ and $v$ and the fracture toughness $K_{\mathrm{IC}}$ of the matrix, the initial crack size $c$, the friction coefficient $\mu$, the parameter $\eta$ and the actual compressive stress $\sigma_{22}$. Thus, the overall instantaneous stiffness tensor $\mathbf{C}^{*}=\mathbf{D}^{*-1}$ can be obtained.

It is interesting to point out that $\mathbf{D}^{*}$ differs from the instantaneous compliance tensor by Basista and Gross (1998), who discussed two alternative simplified crack configurations consisting either of a single crack of length $2 l_{n}$ or of two noninteracting cracks like the ones in Figs 1c, depending on the parameter chosen to drive the loading process. In the second case the formulation leads to the same results by equation (15) at the onset of crack propagation when the size of the wing cracks is much smaller than that of the initial flaw. 


\section{Stability of rectangular orthotropically damaged solids}

In the framework of the classical formulation of the instability theory for elastic solids traced back by Trefftz, the instability of rectangular elastic solids was treated by Kerr and Tang (1967) with regard to isotropic elasticity and by Bazant (1967) in the case of orthotropic elasticity. The bifurcation analysis is based on a classical perturbation technique on the nonlinear equations of the theory of elasticity and assumes the pre-buckling states to be determined by the linearized theory. The equilibrium problem for the adjacent configurations under dead loads leads to the following partial differential equations for the incremental displacements $\dot{u}_{i}(i=1,2)$ from the reference configuration:

$$
\begin{aligned}
& C_{1111}^{*} \dot{u}_{1,11}+\left(C_{1212}^{*}+\sigma_{22}\right) \dot{u}_{1,22}+C_{1212}^{*} \dot{u}_{2,12}+C_{1122}^{*} \dot{u}_{2,21}=0, \\
& C_{1122}^{*} \dot{u}_{1,12}+C_{1212}^{*} \dot{u}_{1,21}+C_{1212}^{*} \dot{u}_{2,11}+\left(C_{2222}^{*}+\sigma_{22}\right) \dot{u}_{2,22}=0,
\end{aligned}
$$

where $C_{i j i j}^{*}(i, j=1,2)$ are the components of the instantaneous stiffness tensor $\mathbf{C}^{*}$.

For the brittle material under consideration, whose behaviour evolves from isotropy at the natural state to orthotropy in damaged states, incremental moduli decrease for increasing compressive stresses. Thus, a lower critical bound for $\sigma_{22}$ may correspond to the emergence of local bifurcation modes associated with the loss of ellipticity of the field equations (17). A nontrivial incremental displacement field of the form $\dot{\boldsymbol{u}}(\boldsymbol{x})=\boldsymbol{v} f(\boldsymbol{g} \cdot \boldsymbol{x})$ ( $\boldsymbol{x}$ and $\boldsymbol{v}$ are the position and displacement vectors; $\boldsymbol{g}$ is a unit vector) may activate at a critical compressive stress $\sigma_{\text {loc }}$. In order to derive $\sigma_{\text {loc }}$, recall that the loss of ellipticity condition is satisfied when the acoustic tensor $\boldsymbol{Q}(\boldsymbol{g})$ related to the differential problem is singular, that is $\operatorname{det}(\boldsymbol{Q}(\boldsymbol{g}))=0$. This turns into an algebraic equation for $g_{i}(i=1,2)$ and $\sigma_{22}$ :

$$
\left[C_{1111}^{*} g_{1}^{2}+\left(C_{1212}^{*}+\sigma_{22}\right) g_{2}^{2}\right]\left[C_{1212}^{*} g_{1}^{2}+\left(C_{2222}^{*}+\sigma_{22}\right) g_{2}^{2}\right]-\left(C_{1212}^{*}+C_{1122}^{*}\right)^{2} g_{1}^{2} g_{2}^{2}=0 .
$$

Equation (18) is simply satisfied by $g_{1}=1, g_{2}=0$ for any compressive stress inducing damage levels characterized by vanishing stiffness components either $C_{1111}^{*}=0$ or $C_{1212}^{*}=0$. Nevertheless, such conditions can be only attained at the onset of unstable crack growth, like the case of splitting failure mode corresponding to $C_{1111}^{*}=0$ and characterized by an incremental displacement field of the type $\dot{u}_{1}=f\left(x_{1}\right), \dot{u}_{2}=0$. Thus, they are not considered here. On the other hand, equation (18) has also solutions explicitly depending on $\sigma_{22}$ if $g_{2} \neq 0$, so that it can be replaced by the following equation for the ratio $r=g_{1} / g_{2}$ :

$$
r^{4}+2 \lambda r^{2}+\rho=0
$$

having defined:

$$
\begin{aligned}
& \lambda=\frac{C_{1111}^{*}\left(C_{2222}^{*}+\sigma_{22}\right)+C_{1212}^{*}\left(C_{1212}^{*}+\sigma_{22}\right)-\left(C_{1212}^{*}+C_{1122}^{*}\right)^{2}}{2 C_{1111}^{*} C_{1212}^{*}}, \\
& \rho=\frac{\left(C_{1212}^{*}+\sigma_{22}\right)\left(C_{2222}^{*}+\sigma_{22}\right)}{C_{1111}^{*} C_{1212}^{*}} .
\end{aligned}
$$

Equation (19) has a trivial solution $\left(g_{1}=0, g_{2}=1\right)$ corresponding to $\rho=0$ for either $\sigma_{22}=-C_{1212}^{*}$ or $\sigma_{22}=-C_{2222}^{*}$. Nontrivial solutions corresponding to $\rho \neq 0$ depend on the specific structure of the instantaneous stiffness tensor $C^{*}$. For the brittle material under consideration, it is easy to prove that equation (19) has at least a real solution but for $\sigma_{22} \leqslant-C_{1212}^{*}$. So, the lower bound for $\sigma_{22}$ due to the loss of ellipticity of the differential equations (17) yields $\sigma_{\text {loc }}=-C_{1212}^{*}$ and the related local bifurcation mode is characterized by an incremental displacement field of the type: $\dot{u}_{1}=f\left(x_{2}\right), \dot{u}_{2}=0$.

Let us now consider the rectangular solid of Fig. 2a, having width $d$ and height $h$, confined between two frictionless platens and subjected to a uniform uniaxial compression $\sigma_{22}$. The related instability problem is governed by equation (17) together with the boundary conditions along the free edges $\left|x_{1}\right|=d / 2$ :

$$
C_{1111}^{*} \dot{u}_{1,1}+C_{1122}^{*} \dot{u}_{2,2}=0 \quad \text { and } \quad \dot{u}_{1,2}+\dot{u}_{2,1}=0,
$$

and along the frictionless simply supported bases $x_{2}=0, x_{2}=h$ :

$$
\dot{u}_{2}=0 \quad \text { and } \quad C_{1212}^{*}\left(\dot{u}_{1,2}+\dot{u}_{2,1}\right)+\sigma_{22} \dot{u}_{1,2}=0 .
$$

The bifurcation analysis is then carried out analysing the emergence of critical conditions corresponding to global buckling associated to non-trivial solutions of the homogeneous boundary value problem in the range $\sigma_{22} \in\left(\sigma_{\text {loc }}, 0\right)$.

In order to do this, the perturbations $\dot{u}_{i}$ are assumed as follows:

$$
\dot{u}_{1}\left(x_{1}, x_{2}\right)=f_{1}\left(x_{1}\right) \cos \left(\gamma x_{2}\right) \quad \text { and } \quad \dot{u}_{2}\left(x_{1}, x_{2}\right)=f_{2}\left(x_{1}\right) \sin \left(\gamma x_{2}\right) \text {, }
$$




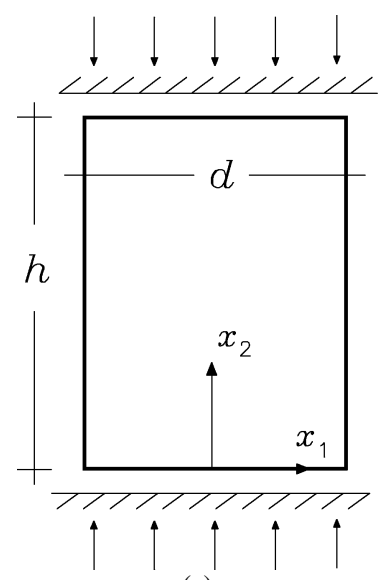

(a)

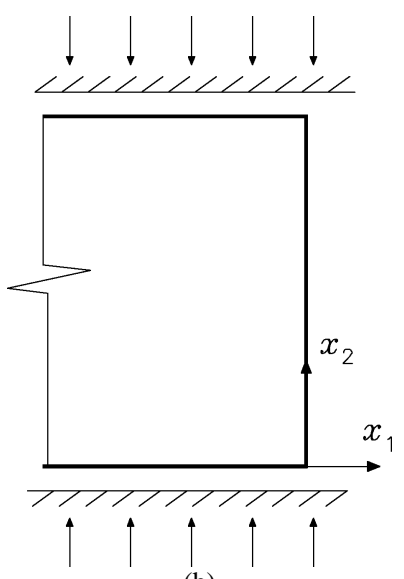

(b)

Fig. 2. Stability of orthotropic solids subjected to a uniform uniaxial compression: (a) rectangular solid; (b) half-space.

where $\gamma=n \pi / h(n \geqslant 1)$, so that the boundary conditions (23) are automatically satisfied. The partial differential equations (17) turn into ordinary differential equations for the functions $f_{1}\left(x_{1}\right)$ and $f_{2}\left(x_{1}\right)$, the primes denoting derivatives with respect to $x_{1}$ :

$$
\begin{aligned}
& C_{1111}^{*} f_{1}^{\prime \prime}+\left(C_{1122}^{*}+C_{1212}^{*}\right) \gamma f_{2}^{\prime}-\left(C_{1212}^{*}+\sigma_{22}\right) \gamma^{2} f_{1}=0, \\
& C_{1212}^{*} f_{2}^{\prime \prime}-\left(C_{1122}^{*}+C_{1212}^{*}\right) \gamma f_{1}^{\prime}-\left(C_{2222}^{*}+\sigma_{22}\right) \gamma^{2} f_{2}=0,
\end{aligned}
$$

whereas the boundary conditions (22) along $\left|x_{1}\right|=d / 2$ become:

$$
C_{1111}^{*} f_{1}^{\prime}+C_{1122}^{*} \gamma f_{2}=0 \text { and } f_{2}^{\prime}-\gamma f_{1}=0 .
$$

Since for the brittle material under consideration it can be proved: $\lambda>0, \rho>0$ and $\lambda^{2}-\rho>0$ for $\sigma_{22}>\sigma_{\text {loc }}$, the system of differential equations (25) has a hyperbolic solution regime given by:

$$
\begin{aligned}
& f_{1}\left(x_{1}\right)=p_{1} \cosh \left(s_{1} \gamma x_{1}\right)+p_{2} \sinh \left(s_{1} \gamma x_{1}\right)+p_{3} \cosh \left(s_{2} \gamma x_{1}\right)+p_{4} \sinh \left(s_{2} \gamma x_{1}\right), \\
& f_{2}\left(x_{1}\right)=t_{1} p_{1} \sinh \left(s_{1} \gamma x_{1}\right)+t_{1} p_{2} \cosh \left(s_{1} \gamma x_{1}\right)+t_{2} p_{3} \sinh \left(s_{2} \gamma x_{1}\right)+t_{2} p_{4} \cosh \left(s_{2} \gamma x_{1}\right),
\end{aligned}
$$

where:

$$
s_{1,2}=\sqrt{\lambda \pm \sqrt{\lambda^{2}-\rho}} \in \Re \quad \text { and } \quad t_{i}=\frac{\left(C_{1212}^{*}+\sigma_{22}\right)-C_{1111}^{*} s_{i}^{2}}{\left(C_{1212}^{*}+C_{1122}^{*}\right) s_{i}} \quad(i=1,2) .
$$

Finally, substitution of equations (27) into the remaining boundary conditions (26) leads to four homogeneous, linear algebraic equations for the constants $p_{i}(i=1,4)$ involving also the stress intensity $\sigma_{22}$. A non-trivial solution exists if and only if the matrix of coefficients is singular. The resulting eigenvalue problem provides the compressive stress $\sigma_{22}$ corresponding to bifurcation as solution of either

$$
\tanh \left(s_{1} \frac{n \pi d}{2 h}\right)-J \tanh \left(s_{2} \frac{n \pi d}{2 h}\right)=0
$$

or

$$
\tanh \left(s_{1} \frac{n \pi d}{2 h}\right)-\frac{1}{J} \tanh \left(s_{2} \frac{n \pi d}{2 h}\right)=0,
$$

where

$$
J=\frac{\left(C_{1111}^{*} s_{2}+C_{1122}^{*} t_{2}\right)\left(1-t_{1} s_{1}\right)}{\left(C_{1111}^{*} s_{1}+C_{1122}^{*} t_{1}\right)\left(1-t_{2} s_{2}\right)} .
$$

Since equation (29) always has real solutions in the range $\left(\sigma_{\text {loc }}, 0\right)$ greater than those satisfying equation (30), the minimum compressive stress $\sigma_{22}$ solving equation (29) and corresponding to $n=1$ is the critical stress $\sigma_{\mathrm{cr}}$ at which the global equilibrium bifurcation is first reached.

Moreover, the emergence of local phenomena associated with surface buckling at the free edges of the specimen is also considered. In order to do this, the instability of the elastic half-space $x_{1} \leqslant 0$ shown in Fig. $2 \mathrm{~b}$ is analysed. For the sake of 
brevity, the details of the approach used, very similar to that just described, can be found in Appendix A. The additional critical stress $\sigma_{\mathrm{cr}}^{\mathrm{s}}$ so derived represents the compressive stress at which a different critical condition may be reached due to surface instability.

To sum up, three critical stresses are derived by the comprehensive bifurcation analysis carried out: $\sigma_{\text {loc }}$ associated with the loss of ellipticity of the field equations, $\sigma_{\mathrm{cr}}^{\mathrm{s}}$ related to surface buckling and $\sigma_{\mathrm{cr}}$ related to global buckling. Some interesting comments can be drawn from comparing such critical stresses. Both $\sigma_{\mathrm{loc}}$ and $\sigma_{\mathrm{cr}}^{\mathrm{s}}$ are independent of the specimen shape. On the contrary, $\sigma_{\mathrm{cr}}$ depends on the specimen shape through the ratio $h / d$ between the height and the width. In general, the following condition is satisfied: $\sigma_{\mathrm{loc}}<\sigma_{\mathrm{cr}}^{\mathrm{s}}<\sigma_{\mathrm{cr}}$. The smaller the ratio $h / d$, the more rapidly $\sigma_{\mathrm{cr}}$ decreases and asymptotically tends to $\sigma_{\mathrm{cr}}^{\mathrm{s}}$.

Finally, it is worth noting that a more complete comprehension of the stability problem here faced should consider the postcritical behaviour and its sensitivity to imperfections. On the other hand, improvements in this sense would require, first, the non-trivial extension of the constitutive model in order to describe microcrack evolution under combinations of compressive and shear stress states, characterizing the post-critical path. Secondly, imperfections apparently are due mainly to non-uniformity of the defect distribution, hence inducing non-uniform stress and strain fields, rather than related to geometrical imperfections.

\section{On the compressive strength of brittle materials}

The damage model described in Section 2 predicts stable responses of brittle materials to uniaxial compressive stress states, so that no limit values of compression to be assumed as the compressive strength can be obtained. However, as a consequence of damage evolution due to microcrack propagation parallel to the applied stress $\sigma_{22}$, the brittle solid stiffness decreases until a critical condition of equilibrium bifurcation may be reached. As found in Section 4, in the case the constraining devices induce an uniform stress state, the critical condition which is first attained corresponds to global buckling. In the framework of a mechanical description of compressive failure, the related value of the critical stress $\sigma_{\mathrm{cr}}$ may be regarded as the compressive strength $\sigma_{\mathrm{c}}$ of the material.

This approach to the compressive failure may result in a nonlinear algebraic system for the compressive stress $\sigma_{22}$ and the damage measure $\zeta$ involving the damage equation and the global buckling equation, respectively:

$$
\zeta=f_{\zeta}\left(\mu, \frac{\sigma_{22}(\pi c)^{1 / 2}}{K_{\mathrm{IC}}}\right), \quad \sigma_{22}=E f_{\mathrm{cr}}\left(\nu, \mu, \frac{K_{\mathrm{IC}}}{E(\pi c)^{1 / 2}}, \eta, \zeta, \frac{h}{d}\right),
$$

where the dependence on the model parameters $\left(E, v, \mu, K_{\mathrm{IC}} / E(\pi c)^{1 / 2}, \eta, h / d\right)$ is emphasized. Then, the compressive stress $\sigma_{22}$ solving equations (32) is assumed as the compressive strength $\sigma_{\mathrm{c}}$ of the specimen.

In Fig. 3 the proposed approach to the physical interpretation of compressive failure is applied to a specimen in plane strain for $\nu=0.2, \mu=0.5, \eta=1$ and $K_{\mathrm{IC}} / E(\pi c)^{1 / 2}=10^{-2}$. In the plane $\left|\sigma_{22}\right| / E$ vs. $\zeta$, the solid curve represents the damage

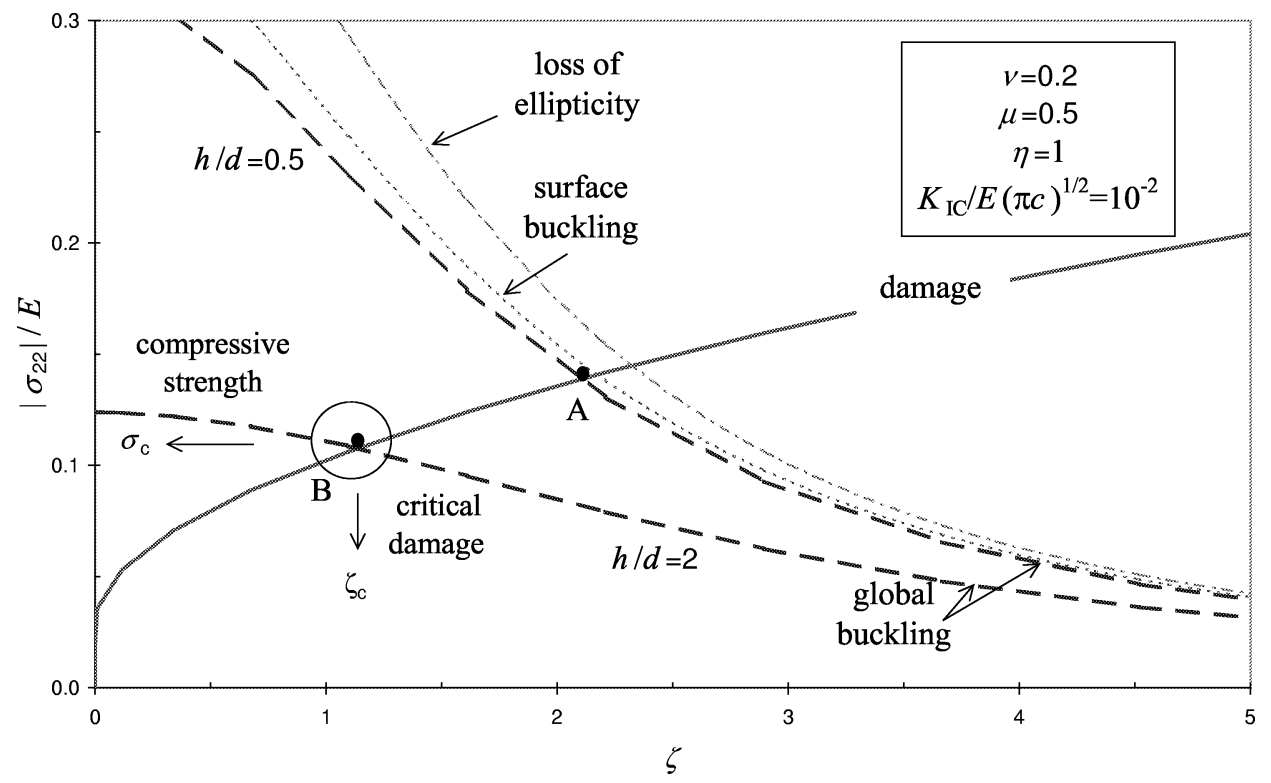

Fig. 3. Compressive strength of rectangular damaged sheets under uniaxial compression. 
equation (32.a), while the dashed ones are referred to the global buckling equation (32.b) for two different values of the specimen shape ratio $(h / d=0.5$ and 2). Points at which such lines cross each other (either $\mathrm{A}$ or $\mathrm{B}$, depending on $h / d)$ are the solutions of system (32), that is the compressive strength $\sigma_{\mathrm{c}}$ and the related damage level $\zeta_{\mathrm{c}}$. In Fig. 3 the diagrams related to surface buckling (dotted line) and loss of ellipticity (dot-dash line) conditions are also shown. As underlined in Section 4, the absolute value of critical stresses $\sigma_{\mathrm{cr}}^{\mathrm{s}}$ corresponding to surface buckling are greater than that of $\sigma_{\mathrm{cr}}$, so that the dotted line lies above every global buckling curve. In addition, increasing the damage level $\zeta$, the buckling curves approach the loss of ellipticity one.

As a consequence of the stability analysis, the approach leads to values of the compressive strength dependent on the specimen geometry (as shown by points A and B in Fig. 3). However, such shape effects are evident when the material is in its natural state, whereas they vanish with increasing damage. At the same time the absolute value of the compressive strength asymptotically approaches the overall instantaneous shear modulus $G^{*}$. The situation is shown in Figs 4 and 5 , where the normalised critical compressive stress is regarded as a function of the ratio $h / d$ (Fig. 4) or of the damage parameter $\zeta$ (Fig. 5) for $v=0.2, \mu=0.5, \eta=1, K_{\mathrm{IC}} / E(\pi c)^{1 / 2}=10^{-2}$ and varying $\zeta$ or $h / d$, respectively.

Furthermore, in Fig. 5 the critical stresses related to global (solid lines) and surface (dashed line) buckling are compared. Hence, it is evident that global buckling occurs before that critical conditions of surface equilibrium bifurcation are reached. On the other hand, the more extensively the damage spreads over the solid, the more the shape effects vanish and the local and global critical conditions of equilibrium bifurcation tend to equal each other.

It can be proved that the calculated compressive strength is much more sensitive to $\eta$ and $K_{\mathrm{IC}} / E(\pi c)^{1 / 2}$ than to the other parameters. To this end, Fig. 6 shows the normalised compressive strength of a specimen in plane strain as a function of $h / d$ for $\nu=0.2, \mu=0.5$ by varying $\eta=1,10,100,1000$ and $K_{\mathrm{IC}} / E(\pi c)^{1 / 2}=10^{-2}, 10^{-3}$. The greater the parameter $\eta$ or the smaller the ratio $K_{\mathrm{IC}} / E(\pi c)^{1 / 2}$, the more rapidly the shape effects vanish. For completeness, some values of the compressive strength independent of the ratio $h / d$ are summarized in table 1 for $\eta=10,100,1000$ and $K_{\mathrm{IC}} / E(\pi c)^{1 / 2}=10^{-3}, 10^{-4}$.

Taking into account that $\eta$ is proportional to the crack density, whereas $K_{\mathrm{IC}} / E(\pi c)^{1 / 2}$ depends on the toughness and Young modulus of the matrix, it follows that the specimen geometry effects on the theoretical compressive strength are more evident for a material consisting of a very tough matrix in which a crack distribution having low density is embedded. On the contrary, even though a stability analysis is carried out, the compressive strength may turn out to be almost independent of the specimen shape if the model parameters are suitably set. As an example, in the case of concrete-like materials one can assume $E=25000 \mathrm{MPa}$ and $v=0.2$ for the matrix, the friction coefficient $\mu=0.5$ and the tensile strength $\sigma_{\mathrm{t}}=2.5 \mathrm{MPa}$. By defining the tensile strength as the applied stress activating mode I unstable crack growth, that is $\sigma_{\mathrm{t}}=K_{\mathrm{IC}} /(\pi c)^{1 / 2}$, the following value of the ratio $K_{\mathrm{IC}} / E(\pi c)^{1 / 2} \approx 10^{-4}$ is obtained. From Fig. 6 and Table 1 note that such a value makes the compressive strength independent of the specimen shape, whatever the parameter $\eta$ is. Moreover, realistic values of the ratio $\left|\sigma_{\mathrm{c}}\right| / \sigma_{\mathrm{t}} \approx 10-12$ can be obtained for $\eta \approx 500$.

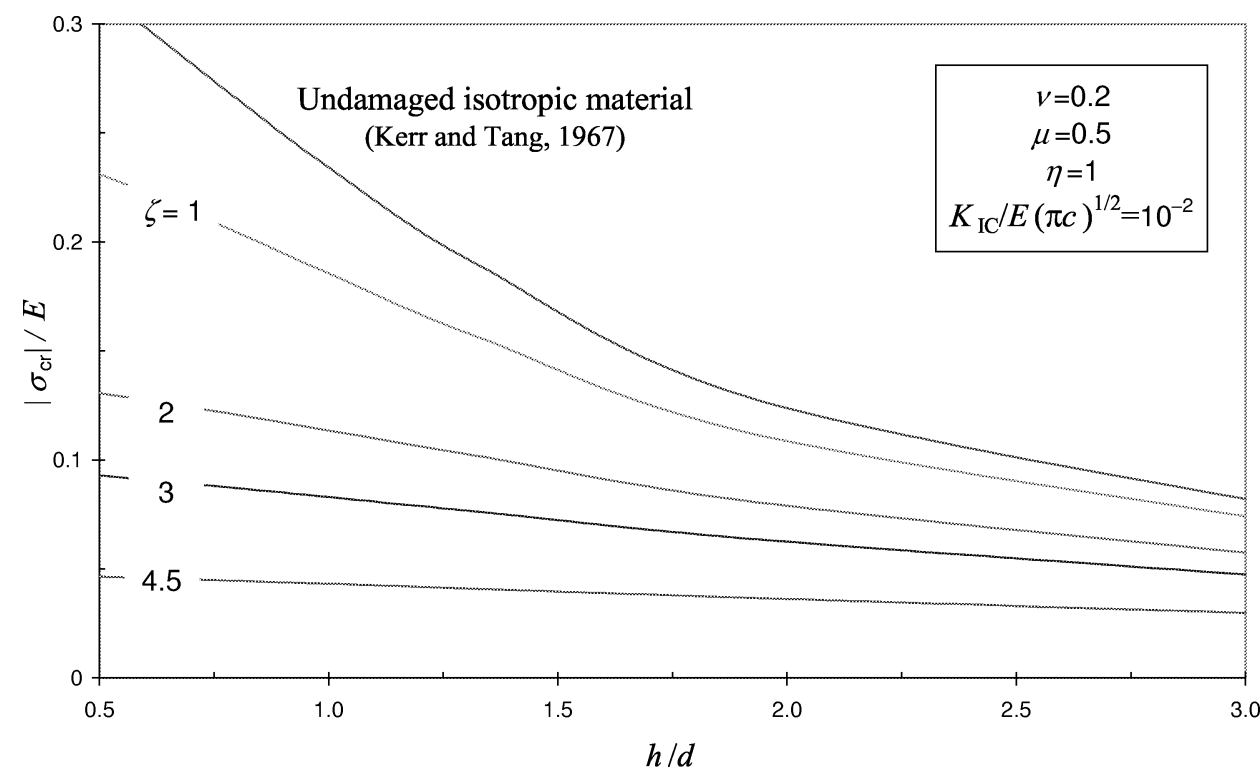

Fig. 4. Shape effects on the critical compressive stress of rectangular damaged sheets. 


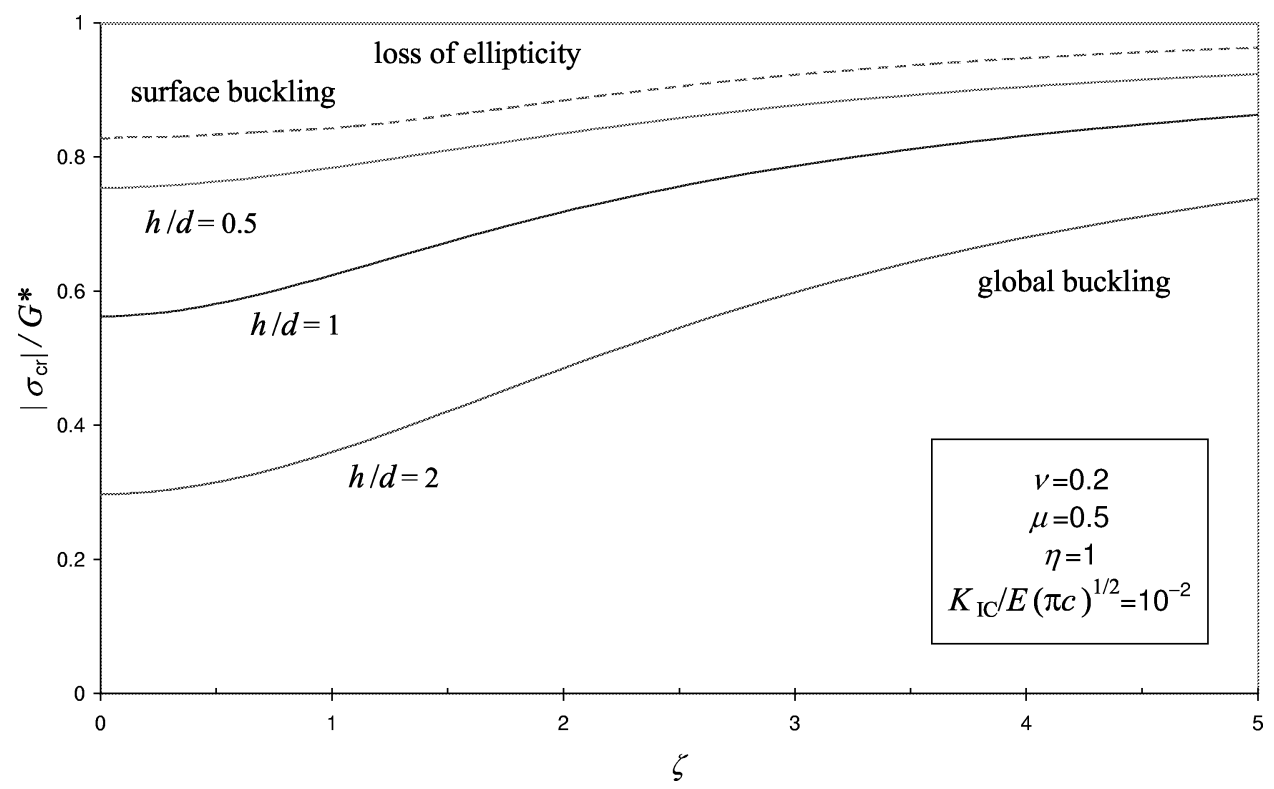

Fig. 5. Damage effects on the critical compressive stress of rectangular damaged sheets.

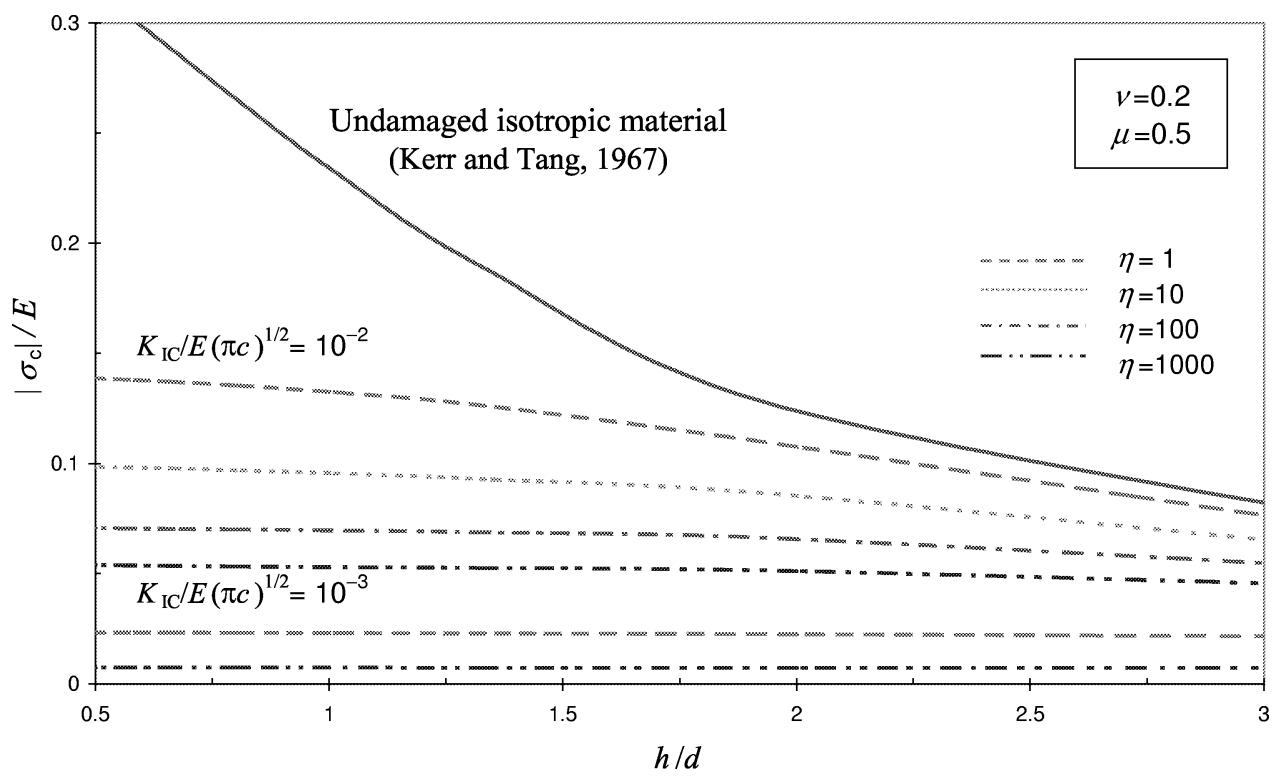

Fig. 6. Compressive strength of rectangular damaged sheets.

Table 1

Constant values of the normalised compressive strength $\left|\sigma_{\mathrm{c}}\right| / E$ for $\nu=0.2$ and $\mu=0.5$.

\begin{tabular}{ccc}
\hline$\left|\sigma_{\mathrm{c}}\right| / E$ & \multicolumn{2}{c}{$K_{\mathrm{IC}} / E(\pi c)^{1 / 2}$} \\
$\eta$ & $10^{-3}$ & $10^{-4}$ \\
\hline 10 & $15 \times 10^{-3}$ & $24 \times 10^{-4}$ \\
100 & $10 \times 10^{-3}$ & $16 \times 10^{-4}$ \\
1000 & $7.5 \times 10^{-3}$ & $10 \times 10^{-4}$ \\
\hline
\end{tabular}


It is worth noting that a shearing critical mode characterised by constant volume is predicted by the model, while splitting failure is observed in experiments carried out by using frictionless platens. In order to explain this apparent contradiction, consider that the limit strength condition associated with incremental shear strain is attained during the compressive loading phase, in which the micromechanical model exhibits extensive dilation due to the inelastic lateral strain. On the other hand, the present model loses its validity in the post-critical phase where damage may no longer be uniformly distributed, so that it is not suitable to describe the kinematics of failure.

\section{Conclusions}

An equilibrium bifurcation approach to the uniaxial compressive failure of microcracked solids undergoing damage is proposed.

The damage process, induced by the applied compressive stress, is predicted by assuming a non planar propagation of noninteracting cracks randomly embedded in an elastic matrix. It follows the evolution of the material response from isotropy at the natural state to orthotropy in damaged states. Since the assumption of non-interacting cracks leads to a stable response of the model, the onset of compressive failure is here interpreted as a bifurcation condition made feasible by the decreasing of stiffness during the damage process. As a consequence, the stability analysis of rectangular orthotropic solids is carried out with reference to the loss of ellipticity of the field equations, surface and global buckling. The minimum critical stress, related to global buckling, is then assumed as the compressive strength of the rectangular sheet. Such theoretical value depends on the matrix moduli $E$ and $\nu$, the friction coefficient $\mu$ between crack faces, the ratio $K_{\mathrm{IC}} / E(\pi c)^{1 / 2}$ involving the matrix toughness and the initial crack size $c$, the parameter $\eta$ related to the initial crack density and the specimen shape ratio $h / d$.

The obtained results show that the greater the parameter $\eta$ or the less tough the matrix, the less the theoretical strength is affected by the specimen shape. Moreover, for a wide range of values of the model parameters, also representative of the concrete-like material response, the theoretical compressive strength turns out to be almost independent of the specimen shape and its absolute value tends towards the actual shear modulus. This circumstance takes place when the damage level makes the difference between global and local instability disappear. In these cases the theoretical compressive strength becomes a material property, in accordance with experimental tests using frictionless devices (van Mier et al., 1997).

Finally, if interaction is taken into account, the damage model predicts a limit compressive stress corresponding to the onset of unstable crack growth (Gambarotta, 1993), to which splitting mode is related. In this frame, compressive failure may be interpreted as the result of a conflict between splitting and buckling. Nevertheless, a comprehensive analysis of the problem, conditioned by the micromechanical damage model and the related parameters, remains an open question.

\section{Acknowledgements}

The present research was carried out with the financial support of the Department for University and Scientific and Technological Research (MURST) in the frame of the Joint Research Project "Structural integrity assessment of large dams".

\section{Appendix A}

The elastic half-space $x_{1} \leqslant 0$ shown in Fig. $2 \mathrm{~b}$, confined between two frictionless platens and subjected to a uniform uniaxial compression $\sigma_{22}$, is here considered. The related instability problem is now governed by equation (17) together with the boundary conditions (23) along the bases $x_{2}=0, x_{2}=h$, whereas conditions (22) are replaced by the following ones:

$$
\begin{aligned}
& C_{1111}^{*} \dot{u}_{1,1}+C_{1122}^{*} \dot{u}_{2,2}=0 \quad \text { and } \quad \dot{u}_{1,2}+\dot{u}_{2,1}=0 \quad \text { at } x_{1}=0, \\
& \dot{u}_{1}=0 \quad \text { and } \dot{u}_{2}=0 \quad \text { for } x_{1} \rightarrow-\infty .
\end{aligned}
$$

Assuming the perturbations $\dot{u}_{i}$ of the form given by equation (24), which automatically satisfy the boundary conditions (23), the problem turns out to be governed by equations (25) for the functions $f_{1}\left(x_{1}\right)$ and $f_{2}\left(x_{1}\right)$, in addition to the boundary conditions (A.1) and (A.2) which become:

$$
\begin{aligned}
& C_{1111}^{*} f_{1}^{\prime}+C_{1122}^{*} \gamma f_{2}=0 \text { and } f_{2}^{\prime}-\gamma f_{1}=0 \quad \text { at } x_{1}=0, \\
& f_{1}=0 \text { and } f_{2}=0 \text { for } x_{1} \rightarrow-\infty
\end{aligned}
$$

Taking into account equation (A.4), the following general solution yields:

$$
\begin{aligned}
& f_{1}\left(x_{1}\right)=q_{1} \exp \left(s_{1} \gamma x_{1}\right)+q_{2} \exp \left(s_{2} \gamma x_{1}\right), \\
& f_{2}\left(x_{1}\right)=t_{1} q_{1} \exp \left(s_{1} \gamma x_{1}\right)+t_{2} q_{2} \exp \left(s_{2} \gamma x_{1}\right),
\end{aligned}
$$


where $s_{i}$ and $t_{i}(i=1,2)$ are defined in equations (28). Finally, substitution of equations (A.5) into the remaining boundary conditions (A.3), leads to a system of homogeneous, linear elastic equations for the constants $q_{i}(i=1,2)$ involving also the stress intensity $\sigma_{22}$. An eigenvalue problem results, so providing the compressive stress which corresponds to bifurcation as solution of the equation:

$$
J=1,
$$

being $J$ given by equation (31). The compressive stress solving equation (A.6) is the critical stress $\sigma_{\mathrm{cr}}^{\mathrm{s}}$ related to surface instability.

\section{References}

Alpa, G., Gambarotta, L., 1993. On the elastic crack interaction in modeling materials. Eng. Fract. Mech. 46, 663-676.

Ashby, M.F., Hallam, S.D., 1986. The failure of brittle solids containing small cracks under compressive stress states. Acta Metall. 34, 497-510. Basista, M., Gross, D., 1998. The sliding crack model of brittle deformation: an internal variable approach. Int. J. Solids Struct. 35, 487-509.

Bazant, Z.P., 1967. L'instabilité d'un milieu continu et la résistance en compression. Bulletin RILEM 35, 99-112.

Bazant, Z.P., Xiang, Y., 1997. Size effect in compression fracture: splitting crack band propagation. J. Eng. Mech. 123, $162-172$.

Biot, M.A., 1965. Mechanics of Incremental Deformations. Wiley, New York.

Brace, W.F., Bombolakis, E.G., 1963. A note on brittle crack growth in compression. J. Geoph. Res. 68, 3709-3713.

Erdogan, F.E., 1962. On the stress distribution in plates with collinear cuts under arbitrary loads, in: Proc. Fourth US National Congress of Applied Mechanics, p. 547.

Fanella, D., Krajcinovic, D., 1988. A micromechanical model for concrete in compression. Eng. Fract. Mech. 29, 49-65.

Gambarotta, L., 1993. Modeling dilatancy and failure of uniaxially compressed brittle materials by microcrack-weakened solids. Eng. Fract. Mech. 46, 381-391.

Horii, H., Nemat-Nasser, S., 1985. Compression-induced microcrack growth in brittle solids: axial splitting and shear failure. J. Geoph. Res. 90, $3105-3125$.

Horii, H., Nemat-Nasser, S., 1986. Brittle failure in compression: splitting, faulting and brittle-ductile transition. Phil. Trans. Roy. Soc. London A 319, 337-374.

Irwin, G.R., 1957. Analysis of stresses and strains near the end of a crack traversing a plate. J. Appl. Mech. - ASME 24, 361.

Kachanov, M., 1992. Effective elastic properties of cracked solids: critical review of some basic concepts. Appl. Mech. Rev. 45, 304-335.

Kemeny, J.M., Cook, N.G.W., 1987. Crack models for the failure of rocks in compression, in: Proc. 2nd Int. Conf. on Constitutive Laws for Engineering Materials, Vol. 2, pp. 879-887.

Kendall, K., 1978. Complexities of compression failure. Proc. Roy. Soc. London A 361, 245-263.

Kerr, A.D., Tang, S., 1967. The instability of a rectangular elastic solid. Acta Mech. 4, 43-63.

Li, S., Lajtai, E.Z., 1998. Modeling the stress-strain diagram for brittle rock loaded in compression. Mech. Mat. 30, $243-251$.

Myer, L.R., Kemeny, J.M., Zheng, Z., Suarez, R., Ewy, R.T., Cook, N.G.W., 1992. Extensile cracking in porous rock under differential compressive stress. Appl. Mech. Rev. 45, 263-280.

Moss, W.C., Gupta, Y.M., 1982. A constitutive model describing dilatancy and cracking in brittle rocks. J. Geophys. Res. 87, $2985-2998$.

Nemat-Nasser, S., Hori, M., 1993. Micromechanics: Overall Properties of Heterogeneous Materials. Elsevie, Amsterdam.

Nemat-Nasser, S., Obata, M., 1988. A microcrack model of dilatancy in brittle materials. J. Appl. Mech. 55, $24-35$.

Steif, P.S., 1984. Crack extension under compressive loading. Eng. Fract. Mech. 20, 463-473.

Tada, H., Paris, P.C., Irwin, G.R., 1973. The Stress Analysis of Cracks Handbook. Paris Production Incorporated, St Louis, USA.

van Mier, J.G.M., Shah, S.P., Arnaud, M., Balayssac, J.P., Bascoul, A., Choi, S., Dasenbrock, D., Ferrara, G., French, C., Gobbi, M.E., Karihaloo, B.L., König, G., Kotsovos, M.D., Labuz, J., Lange-Kornbak, D., Markeset, G., Pavlovic, M.N., Simsch, G., Thienel, KC., Turatsinze, A., Ulmer, M., van Geel, H.J.G.M., van Vliet, M.R.A., Zissopoulos, D., 1997. Strain-softening of concrete in uniaxial compression. Mater. Struct. 30, 195-209.

Zaitsev, Y.V., Wittmann, F.H., 1981. Simulation of crack propagation and failure of concrete. Mater. Struct. 14, 357-365.

Zhan, S., Wang, T., Han, X., 1999. Analysis of two-dimensional finite solids with microcracks. Int. J. Solids Struct. 36, $3735-3753$. 